\section{Ethnologie française}

Février 2020 Tome $\mathrm{L} \mathrm{n}^{\circ} 1$

\section{TRAITES \\ ESCLAVAGISTES \\ ET MÉMOIRE CULTURELLE}

Hommage - Suzanne Tardieu-Dumont (1920-2019) par Martine Segalen

ÉDITEUR INVITÉ : Gaetano Ciarcia

\section{- Gaetano Ciarcia}

Introduction. Visions du passé et mémoire culturelle des traites esclavagistes

- Renaud Hourcade

La politique des excuses. Repentir officiel et gestion stratégique de la culpabilité dans un ancien port négrier (Liverpool)

- Francesca Cozzolino

Aux bords de la critique et de la réconciliation. Controverses mémorielles à Bordeaux : le projet artistique «Les Revenants »

- Lawrence Aje

La commémoration de l'esclavage dans l'espace public aux Etats-Unis : une amnésie sélective?

- Rossila Goussanou

Visites touristiques et détournements du passé sur la Route de l'Esclave à Ouidah

- Martin Mourre

Histoire et émotions. Mise en scène de la Maison aux Esclaves à Gorée au Sénégal

- Jérôme Souty

La gestion du passé de l'esclavage depuis le vieux port de Rio de Janeiro (2010-2019). Politiques publiques, réappropriations locales, paysage de mémoire

- Marie-Aude Fouéré

Muséifier la traite de l'esclavage à Zanzibar. Vérité, contre-vérité et incertitude au marché aux esclaves

- Gaetano Ciarcia

Instituer le souvenir de l'esclavage en Guadeloupe et à Nantes : les contentieux du don commémoratif

- Gaetano Ciarcia et Lucile Combreau

L'opaque éclat du passé de l'esclavage

Entretien avec Nicola Lo Calzo autour du projet Cham

\section{LECTURES SUR LE THÈME}

\section{VARIA}

- Marianne Lemaire

Age, sexe et génération de l'ethnologue. Denise Paulme en

Côte d'Ivoire dans les années 1960

- Frédéric Fruteau de Laclos

Quand agir, c'est penser. Genres de vie et genres de connaissance dans l'anthropologie d'André Varagnac

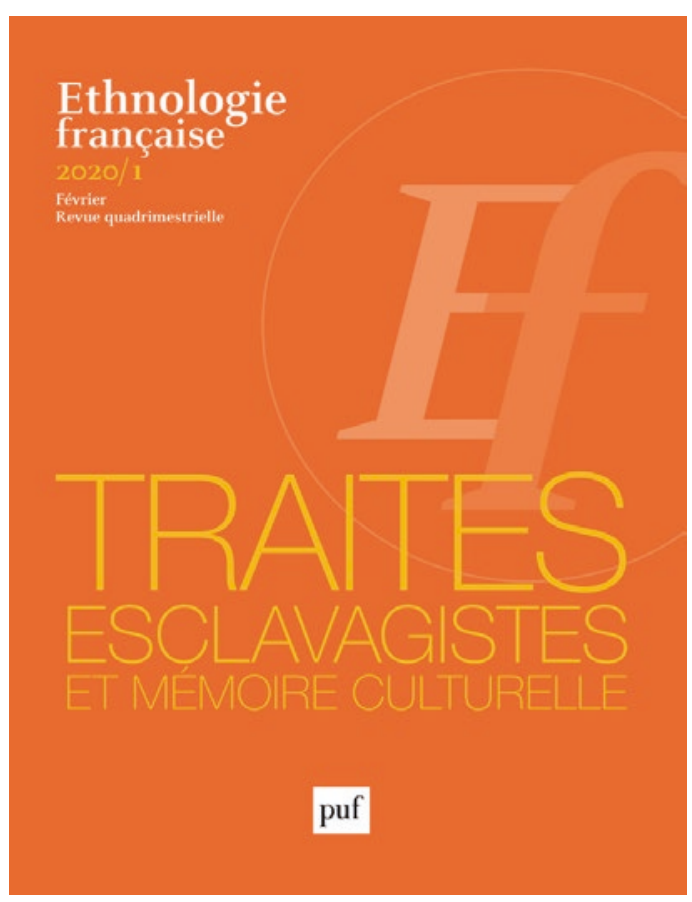

$\mathrm{D}$ epuis quelques décennies, le passé de l'esclavage est devenu une question sociétale importante et dont la charge politique est considérable. Issus de recherches concernant des contextes africains, américains et européens, les articles ici réunis interrogent les gestes, les récits et les visions propres à l'institution globalisée et plurielle d'une mémoire culturelle des traites négrières. Une telle mémoire est à l'œuvre dans la création de lieux, de monuments, de rituels ou de discours dont la quête de consensus ou de réparation n'est pas sans soulever, parfois, de vives polémiques et de fortes tensions.

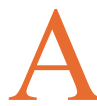
insi, ce dossier vise à montrer les manières dont le souvenir du passé esclavagiste se manifeste également par le biais de postures morales, de revendications identitaires et d'émotions. Analyser les enjeux sous-jacents de reconnaissance, d'héritage, de dette qui gouvernent ces fabrications mémorielles est l'un des objectifs de cette livraison.
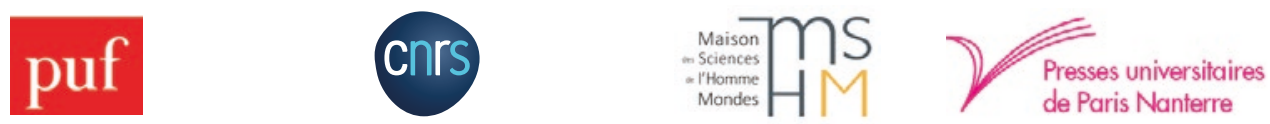

Maison des Sciences de l'Homme Mondes

21, avenue de l'Université - 92023 Nanterre cedex

0146692663 - ethnologie.francaise $@$ mae.u-paris10.fr

$22 €$ le numéro en librairie ou aux Presses Universitaires de France

en ligne http://www.cairn.info/revue-ethnologie-francaise.htm 


\section{Instructions aux auteurs}

Les Annales n'acceptent que les travaux originaux et inédits, rédigés en français, en anglais, en italien, en allemand ou en espagnol. Tout manuscrit proposé est soumis au comité de rédaction dans un délai de six mois et fait l'objet d'une évaluation par deux experts extérieurs. Le comité peut ensuite décider d'accepter le manuscrit en l'état ou de l'accepter sous réserve de modifications, ou encore demander une deuxième version qui sera de nouveau évaluée ou, enfin, refuser directement l'article.

Les manuscrits doivent être envoyés à l'adresse annales@ehess.fr dans un format de type Word ou Open Office (et non en format PDF). Ils sont accompagnés d'un résumé en français et en anglais (précédé de son titre traduit) d'environ 1500 signes. Les documents iconographiques sont remis dans des fichiers séparés, en haute définition et dans une dimension suffisante par rapport au format de la revue $(170 \mathrm{~mm} \times 240 \mathrm{~mm})$. Ils sont accompagnés de titres, de légendes et de la mention des sources. La nationalité des auteurs ainsi que leur appartenance institutionnelle doivent être précisées.

Le texte doit être présenté en double interligne et suivre nos recommandations disponibles à l'adresse http://annales.ehess.fr/index. php?497. Il ne doit pas dépasser les 90000 signes (notes et espaces compris), les notes en bas de page sont limitées à 100. Les citations en langue étrangère sont traduites, l'original est donné éventuellement en note.

Nous attirons l'attention des auteurs sur le fait que leur article, une fois accepté pour publication, sera traduit en anglais pour une diffusion papier et en ligne. Les auteurs sont mis en contact direct avec les traducteurs, ce dialogue favorisant la réalisation d'une traduction scientifique de qualité.

Les auteurs sont informés qu'il leur revient de trouver, au préalable, les versions originales anglaises des citations qu'ils fournissent en français dans leur article, lorsqu'il en existe une édition canonique. Ils s'engagent à fournir un fichier de ces versions anglaises, contenant aussi les nouvelles références éditoriales, les versions anglophones des noms propres cités, ainsi que celles des concepts employés; ce fichier sera transmis au traducteur le moment venu. 


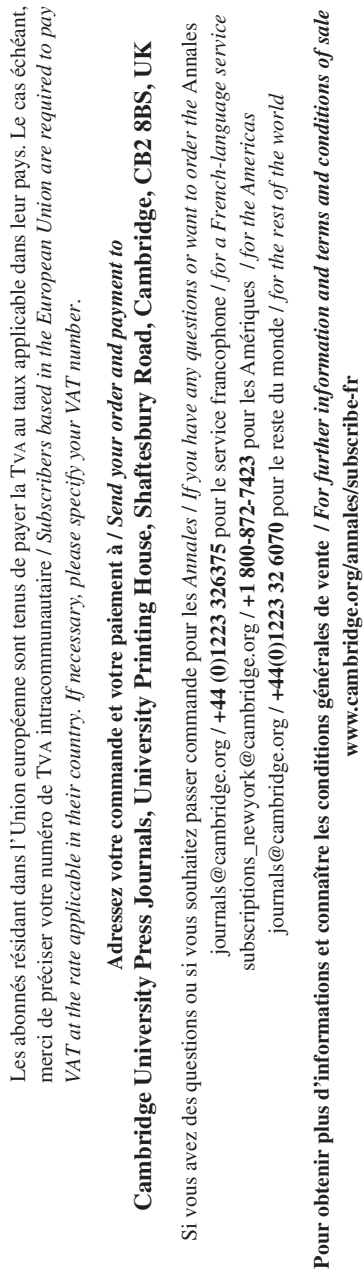


Tous droits de traduction, d'adaptation et de reproduction par tous procédés, réservés pour tous pays.

Le Code de la propriété intellectuelle (CPI) n'autorisant, aux termes de l'article L. 122-5, $2^{\circ}$ et $3^{\circ}$ a, d'une part, que les «copies ou reproductions strictement réservées à l'usage privé du copiste et non destinées à une utilisation collective» et, d'autre part, que les analyses et les courtes citations dans un but d'exemple et d'illustration, «toute représentation ou reproduction intégrale ou partielle faite sans le consentement de l'auteur ou de ses ayants droit ou ayants cause est illicite» (art. L. 122-4 du CPI). Cette représentation ou reproduction, par quelque procédé que ce soit, constituerait donc une contrefaçon sanctionnée par les articles L. 335-2 et suivants du CPI.

Diffusion Cambridge University Press (Cambridge/New York) — Dépôt légal : $2019-\mathrm{n}^{\mathrm{o}} 1$, janvier-mars 2019

Imprimé au Royaume-Uni par Bell and Bain Ltd. ISSN (édition française): 0395-2649 (version imprimée) et 1953-8146 (version numérique)

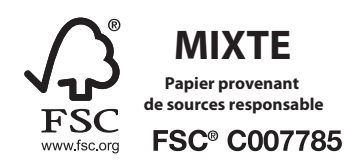




\section{$\mathrm{N}^{\circ} 2$ \\ à paraître \\ avril-juin 2019}

Historiographie transnationale

Renaud Soler

Tom Stammers

Démocratie athénienne

Mirko Canevaro

Vincent Azoulay

Historiographie (comptes rendus)

Résumés / Abstracts

Livres reçus

ISBN 978-2-7132-2775-2

$g=\left\|_{782713}\right\|_{227752}$ 